\title{
MiR-124 inhibits invasion and induces apoptosis of ovarian cancer cells by targeting programmed cell death 6
}

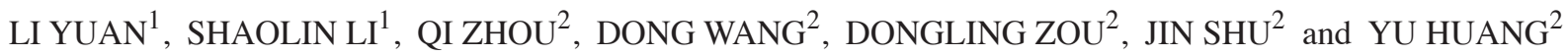 \\ ${ }^{1}$ Department of Radiological Medicine, Chongqing Medical University, Chongqing 400016; \\ ${ }^{2}$ Department of Gynecologic Oncology, Chongqing Cancer Institute, Chongqing 400030, P.R. China
}

Received March 13, 2016; Accepted July 14, 2017

DOI: $10.3892 / \mathrm{ol} .2017 .7157$

\begin{abstract}
Epithelial ovarian cancer remains the most common type of malignant tumor of the female reproductive system worldwide. Routine surgery and chemotherapy are the best treatments available for patients with ovarian cancer; however, almost $40 \%$ of ovarian cancer cases are intractable, with poor 5-year survival rates. MicroRNAs (miRNA) are endogenous small non-coding RNA molecules that function in transcriptional and post-transcriptional regulation of gene expression in various cellular processes. Recent studies demonstrated that microRNA (miR)-124 was downregulated in numerous types of tumors; however, the function and mechanism underlying miR-124 in epithelial ovarian cancer remain unclear. The present study revealed that miR-124 may be significantly downregulated in epithelial ovarian cancer. Using prediction algorithms and luciferase reporter gene assays, the present study identified and confirmed programmed cell death 6 (PDCD6) as a novel, direct target of miR-124. Overexpression of miR-124 suppressed PDCD6 expression, inhibited cell proliferation, migration and invasion, and induced apoptosis in SKOV3 and OCVAR3 cells in vitro. In the present study, overexpression of PDCD6 in epithelial ovarian cancer cells co-transfected with miR-124 effectively reversed the miR-124-induced apoptosis. Therefore, the results of the present study suggested that miR-124 is a tumor suppressor miRNA and a potential target for future treatment of ovarian malignant neoplasms.
\end{abstract}

\section{Introduction}

MicroRNAs (miRNAs) are a class of endogenous non-coding small RNAs molecules that trigger transcriptional or post-transcriptional regulation by binding to the 3'-untranslated region (UTR) of target genes (1-3). In recent studies,

Correspondence to: Professor Shaolin Li, Department of Radiological Medicine, Chongqing Medical University, 1 Yixueyuan Road, Chongqing 400016, P.R. China

E-mail: cqmu_lishaolin@sina.com

Key words: ovarian cancer, miR-124, programmed cell death 6, SKOV3, OCVAR3, microRNA, metastasis, cell migration, apoptosis
miRNAs were revealed to function as either oncogenes or tumor suppressor genes in biological processes, including cell proliferation, migration, apoptosis and differentiation and in tumorigenesis of a number of types of human cancer (3-7). In females worldwide, ovarian cancer is a common gynecological malignancy and remains a major problem, with high mortality due to diagnosis at an advanced stage and distant metastases $(8,9)$. Various miRNAs are post-transcriptionally deregulated in ovarian cancer (10-13). Although evidence has supported miRNAs as a potential target in cancer diagnosis, therapy and prognosis, the role of miRNAs in ovarian cancer remains unclear. miR-124, a tumor suppressor miRNA, is significantly downregulated in a number of human malignant tumors (14-17), including hepatocellular carcinoma and gastric carcinoma; however, the possible effect of miR-124 in ovarian cancer has not been fully explored.

Programmed cell death 6 (PDCD6) is a calcium-binding protein that regulates cell proliferation and death and belongs to the penta-EF-hand protein family $(18,19)$. Previous studies have indicated that PDCD6 is critical in T-cell receptor-, Fasand glucocorticoid-induced programmed cell death $(19,20)$. Furthermore, PDCD6 was demonstrated to be a pro-apoptotic protein involved in cell viability functions $(19,21)$; however, its role in regulating migration and invasion in ovarian cancer is unclear.

The present study aimed to determine whether miR-124 and PDCD6 are involved in the apoptosis, migration and invasion of ovarian cancer. It was revealed that miR-124 was downregulated in ovarian cancer cell lines and revealed an association between miR-124 expression level and ovarian cancer cell metastasis. The present study further identified PDCD6 as a novel, direct target of miR-124 that suppressed apoptosis in ovarian cancer progression, and miR-124 rescue experiments restored PDCD6 expression level. The results of the present study demonstrated that miR-124 suppressed cell motility, migration and invasion and induced cell apoptosis by downregulating PDCD6. Thus, miR-124 may provide a novel target for effective treatment of ovarian cancer.

\section{Materials and methods}

Patients and tissue samples. Human ovarian tumor tissues were collected from the 30 patients who had ovarian epithelial carcinoma and non-neoplastic ovarian tissues from 30 healthy 
individuals under 60 years of age who received surgical resection between January 2009 and December 2012 at the Department of Gynecologic Oncology at Chongqing Cancer Hospital (Chongqing Cancer Institute, Chongqing, China). The present study was approved by the Ethics Committee of the Chongqing Cancer Hospital (Chongqing Cancer Institute, Chongqing, China). Written informed consent was provided by all patients prior to enrollment in the present study.

Cell lines and cell culture. SKOV3 and OVCAR3 human ovarian cells were purchased from the Cell Bank of the Chinese Academy of Sciences (Shanghai, China). All cells were cultured in RPMI-1640 medium (Gibco; Thermo Fisher Scientific, Inc., Waltham, MA, USA) supplemented with $10 \%$ fetal bovine serum (FBS; Hyclone, Logan, UT, USA) in a humidified atmosphere of $5 \% \mathrm{CO}_{2}$ at $37^{\circ} \mathrm{C}$.

Transient transfection of miR-124. The miR-124 mimics and the miR-124 mimics scramble were synthesized by Shanghai GeneChem Co., Ltd. (Shanghai, China). The sequences (5'-3') were as follows: hsa-miR-124-5p mimics forward, 5'-CGUGUU CACAGCGGACCUUGAU-3' and reverse, 5'-AUCAAGGUC CGCUGUGAACACG-3'; miR-124 mimics scramble forward, 5'-UUCUCCGAACGUGUCAGU-3' and reverse, 5'-ACG UGACACGUUCGGAGAA-3'. The pcDNA3.1/PDCD6 and pcDNA3.1/control were synthesized by Guangzhou RiboBio Co., Ltd. (Guangzhou, China). Cells were harvested and plated in six-well plates at a density of $5 \times 10^{5}$ cells $/ \mathrm{ml}$. All cell transfections were performed using Lipofectamine 2000 reagent (Invitrogen; Thermo Fisher Scientific, Inc.), according to the manufacturer's instructions. In the rescue experiment, cells were co-transfected with miR-124 mimics and plasmids.

RNA isolation and reverse transcription-quantitative polymerase chain reaction ( $R T-q P C R)$. Total RNA was extracted using TRIzol ${ }^{\circledR}$ reagent (Invitrogen; Thermo Fisher Scientific, Inc.) from clinical tumor samples and transfected cells according to the manufacturer's instructions. RNA concentration and quality were evaluated and $0.5 \mu \mathrm{g}$ RNA was used for RT. MMLV reverse transcriptase (Takara Bio., Inc., Otsu, Japan) was used to synthesize complementary DNA (cDNA). For synthesis of miR-124 cDNA, a miR-124 RT primer was used and U6 small nuclear RNA was used as the internal control. cDNA for other target genes was amplified using the primer oligo(dT) with $\beta$-tubulin as the internal control. Target genes and controls were analyzed by RT-qPCR using SYBR Premix Ex Taq (Promega Corporation, Madison, WI, USA). The following primers were used for qPCR: miR-124 forward, 5'-TGCGGTAAGGCACGCGGTG-3' and reverse, 5'-CCA GTGCAGGGTCCGAGGT-3'; U6 forward, 5'-TGCGGGTGC TCGCTTCGGCAGC-3' and reverse, 5'-CCAGTGCAGGGT CCGAGGT-3'; PDCD6 forward, 5'-TCCAGAGGGTCGATA AAGACA-3' and reverse, 5'-TTCTGCCAGTCCGTGATG T-3'; $\beta$-tubulin forward, 5'-TTGGCCAGATCTTTAGACCAG ACAAC-3'; and reverse, 5'-CCGTACCACATCCAGGACAGA ATC-3'. PCR was conducted at $94^{\circ} \mathrm{C}$ for $4 \mathrm{~min}$, followed by 40 cycles of $94^{\circ} \mathrm{C}$ for $30 \mathrm{sec}, 58^{\circ} \mathrm{C}$ for $30 \mathrm{sec}$ and $72^{\circ} \mathrm{C}$ for $30 \mathrm{sec}$ in an iQ5 Real-time PCR system (Bio-Rad Laboratories, Inc., Hercules, CA, USA). All the samples were assessed by relative quantification (2- ${ }^{-\Delta \Delta \mathrm{Cq}}$ method) (22).
Target prediction. The prediction of the PDCD6 3'-UTR as a miR-124 binding target was determined using TargetScan (www.targetscan.org) and PicTar (www.pictar.org).

Dual-luciferase reporter assay. A luciferase reporter assay was used to monitor the direct interaction between the miRNA and its target mRNA. The binding site of miR-124 in the 3'-UTR of the target mRNA was cloned into the pmirGLO Dual-Luciferase miRNA Target Expression Vector (Promega Corporation), according to the manufacturer's instructions. Cells were subsequently co-transfected with miRNA and the wild type or mutant 3'-UTR for the luciferase assay. Following transfection for $48 \mathrm{~h}$ at room temperature, the cells were harvested for protein extraction. Luciferase intensity was examined using the Dual Luciferase Reporter Gene Assay kit (Beyotime Institute of Biotechnology, Haimen, China), according the manufacturer's protocol. Renilla luciferase intensity was used an internal control.

Cell migration and invasion assays. Cell migration was evaluated using a wound-healing assay. At $24 \mathrm{~h}$ after transfection, a wound was created by $20 \mu \mathrm{l}$ pipette tips. in all culture wells. Cultures were imaged at 0,24 and $48 \mathrm{~h}$ to assess cell migration. Light microscopy was undertaken at x100 magnification at 0,24 and $48 \mathrm{~h}$ to assess cell migration.

The present study used a Transwell assay with a Matrigel-coated membrane matrix (BD Biosciences, Franklin Lakes, NJ, USA) to assess cell invasion. After transfection, cells $\left(5 \times 10^{4}\right)$ were seeded in the upper chamber of a Millicell Transwell chamber (EMD Millipore, Billerica, MA, USA) with $200 \mu 1$ RPMI-1640 medium without FBS. Cells that migrated through the membrane were fixed with $4 \%$ methanol for $10 \mathrm{~min}$ at room temperature. and stained with $0.1 \%$ crystal violet for $15 \mathrm{~min}$ at room temperature. Cells were then imaged at x100 magnification and counted using an inverted microscope.

Cell survival assay. SKOV3 and OVCAR3 cells were plated onto 96-well plates $48 \mathrm{~h}$ after transfection at a density of $5 \times 10^{4}$ cells $/ \mathrm{ml}$. A total of three replicate wells were examined for each group. When cells reached 60-70\% confluency, $10 \mu \mathrm{l}$ $5 \mathrm{mg} / \mathrm{ml}$ MTT was added to each well. After $4 \mathrm{~h}$, the culture medium was discarded, $100 \mu \mathrm{l}$ dimethyl sulfoxide was added to each well and the plates were agitated at room temperature for $1 \mathrm{~h}$. Absorbance (optical density) was evaluated using a microplate reader at a wavelength of $490 \mathrm{~nm}$. This experiment was repeated three times.

Western blot analysis. At the indicated time, SKOV3 and OVCAR3 whole-cell lysates were extracted using radioimmunoprecipitation assay buffer and incubated for $30 \mathrm{~min}$ on ice. Cell lysates were prepared from cells seeded on 6-well plates at $10^{6}$ cells per well using radioimmunoprecipitation assay buffer (10 mM Tris-HCl, pH 7.4, 1\% Triton X-100, 0.1\% SDS, $1 \%$ NP- 40, $1 \mathrm{mM} \mathrm{MgCl2}$ ) containing protease inhibitors at $48 \mathrm{~h}$ post-transfection.

Proteins were quantified using the BCA method, according to the manufacturer's instructions (Thermo Fisher Scientific, Inc.). Proteins $(10 \mu \mathrm{g})$ were separated by $10 \%$ SDS-PAGE and then transferred to polyvinylidene difluoride membranes 
A

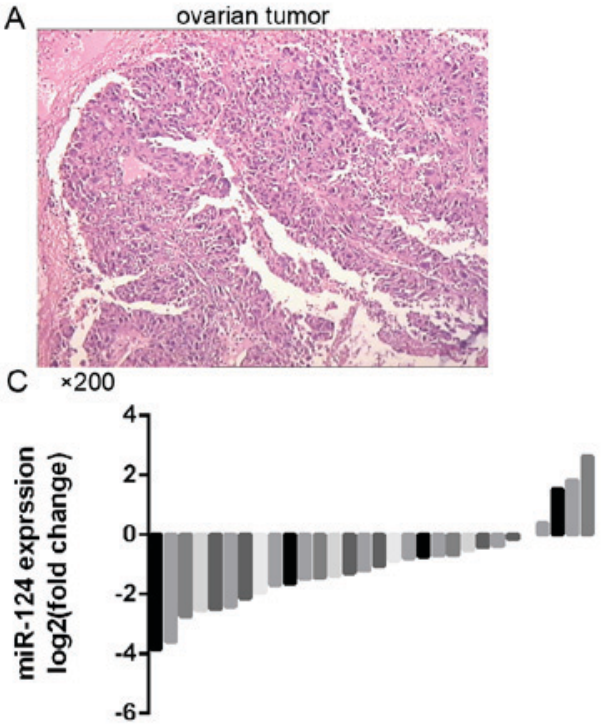

B
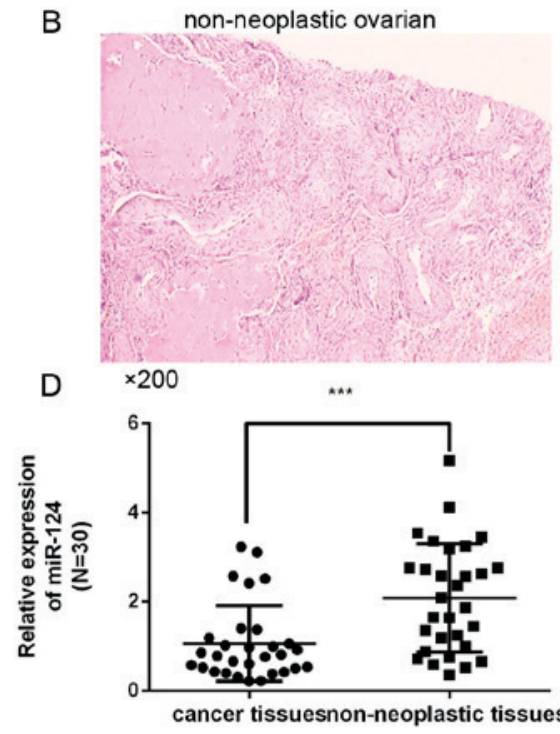

E

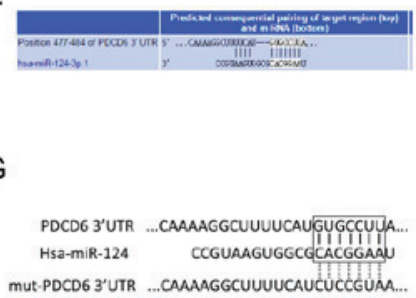

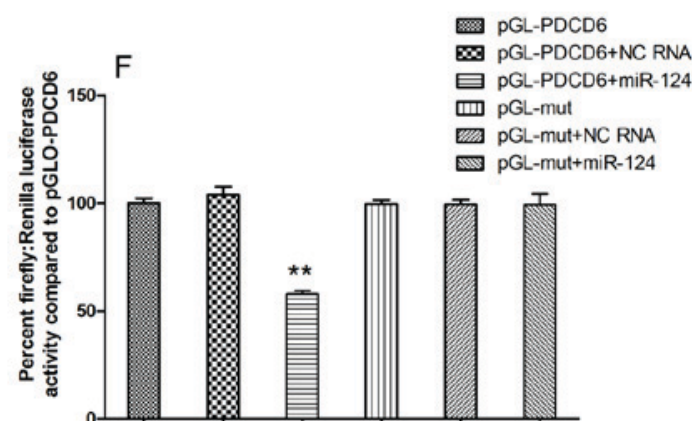

Figure 1. Expression level of miR-124 in human ovarian tissues and PDCD6 is a target of miR-124. (A) Representative hematoxylin and eosin staining of ovarian tumor tissue specimens (magnification, x200). (B) Representative hematoxylin and eosin staining of non-neoplastic ovarian tissue specimens (magnification, x200). (C) Expression of miR-124 was detected in 30 human ovarian cancer tissues by polymerase chain reaction. Data are presented as log2 values of the change in ovarian tumor tissues relative to non-neoplastic ovarian tissues. (D) The expression of miR-124 in ovarian cancer tissues was decreased compared with in non-neoplastic ovarian tissues $\left.{ }^{\left({ }^{* *} \mathrm{P}\right.} \mathrm{P}<.001\right)$. (E) The putative association between PDCD6 and miR-124 was revealed by TargetScan. $(\mathrm{F}) \mathrm{The}$ dual-luciferase reporter assay revealed regulation of PDCD6 by miR-124, including wild-type PDCD6 or mutant PDCD6 3'-UTR transfected with or without miR-124 mimics or scramble. (G) Diagram of the PDCD6 3'-UTR with potential binding-sites for miR-124. The data from three in vitro independent experiments are presented ( $(*$ P $<0.01)$. NC, negative control; miR, microRNA; UTR, untranslated region; mut, mutant; PDCD6, programmed cell death 6.

(EMD Millipore). The membranes were blocked in 5\% non-fat milk/TBST for $1 \mathrm{~h}$ at room temperature. Thereafter, they were incubated with rabbit monoclonal anti-PDCD6 antibody $(1: 1,000 ;$ ab133326) and anti- $\beta$-tubulin antibody $\left(1: 1,000 ;\right.$ ab6046) (Abcam, Cambridge, UK) overnight at $4^{\circ} \mathrm{C}$. After adding peroxidase-conjugated goat anti-rabbit antibody (1:5,000; BA1055; Boster Biological Technology, Pleasanton, CA, USA) for $1 \mathrm{~h}$ at room temperature, the membranes were visualized by Western Lightning ${ }^{\circledR}$-ECL, Enhanced Chemiluminescence Substrate (PerkinElmer, Inc., Waltham, MA, USA, and X-ray film was applied to analyze the image and intensity of band.

Cell cycle and apoptosis assays. At $48 \mathrm{~h}$ after transfection, $1 \times 10^{6}$ cells were seeded onto $75-\mathrm{mm}$ dishes. To analyze the cell cycle stage, the cells were harvested, by trypsin and washed twice with cold PBS, fixed in ice-cold 70\% ethanol and stained with $100 \mu \mathrm{g} / \mathrm{ml}$ propidium iodide (Sigma-Aldrich; Merck KGaA, Darmstadt, Germany) supplemented with $1 \mathrm{mg} / \mathrm{ml} \mathrm{RNase}$ A for $30 \mathrm{~min}$ at room temperature in the dark. Subsequently, the cell cycle stages were analyzed using a flow cytometer and Cell Quest software version 3.3 (BD
Biosciences). To analyze apoptosis, the cells were harvested from the culture dishes by trypsinization. After washing twice with cold PBS, cells were resuspended in $200 \mu 1$ binding buffer and incubated with $10 \mu 1$ Annexin V-R-PE and $10 \mu 1$ 7-ADD (SouthernBiotech, Birmingham, AL, USA) in the dark at $4^{\circ} \mathrm{C}$ for $30 \mathrm{~min}$. Thereafter, $380 \mu \mathrm{l}$ binding buffer was added to each tube and the samples were analyzed by flow cytometry.

Statistical analysis. All data were presented as the mean \pm standard deviation. Error bars represent standard error. One-way analysis of variance, followed by Fisher's least significance test or two-tailed Student's t-test were used to analyzed the statistical significance. $\mathrm{P}<0.05$ was considered to indicate a statistically significant difference.

\section{Results}

miR-124 is downregulated in ovarian cancer. To analyze the expression level of miR-124 in ovarian cancer progression, RT-qPCR was performed using TaqMan probes. Ovarian cancer tissue and normal ovarian tissue have distinct 

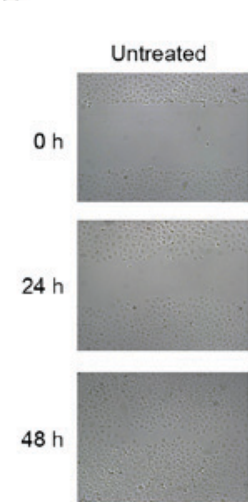

C

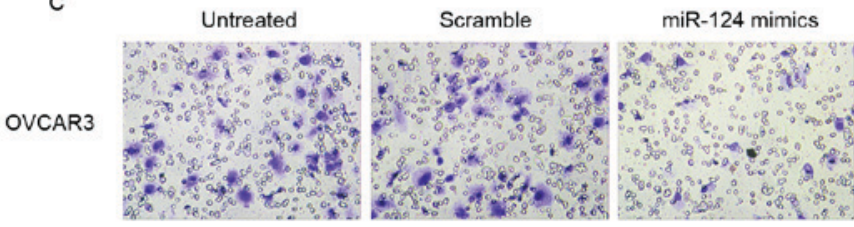

E

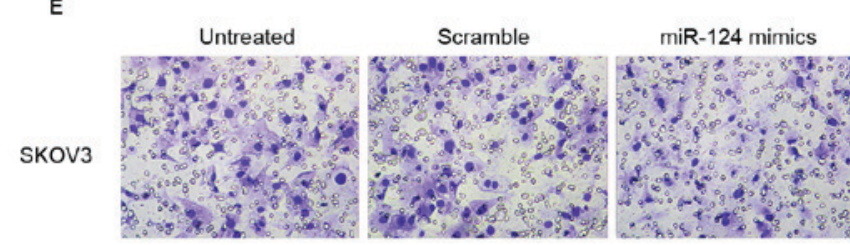

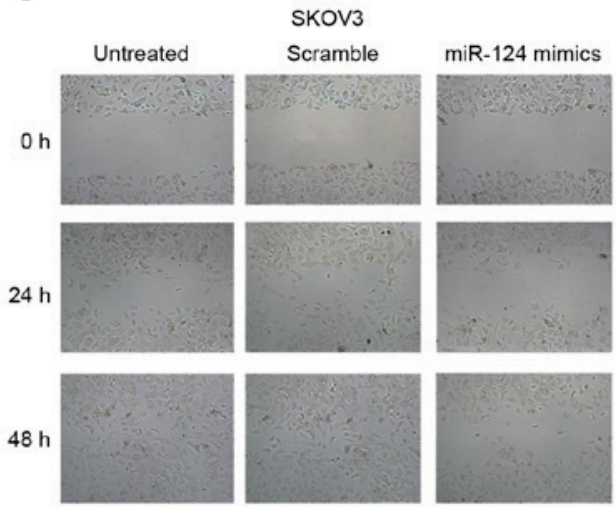

D
Untreated

miR-124 mimics

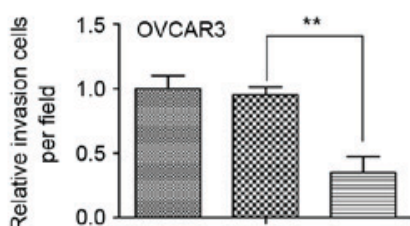

$\mathrm{F}$
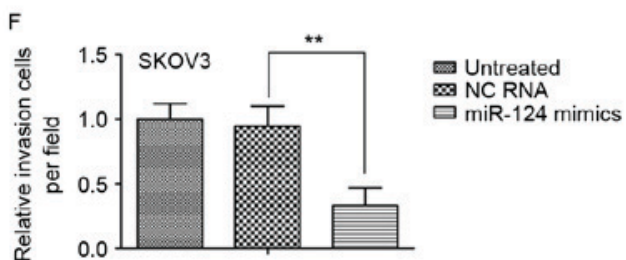

Figure 2. Overexpression of miR-124 suppressed ovarian cancer cell migration and invasion. (A) OCVAR3 and (B) SKOV3 cells transfected or not with miR-124 mimics or scramble for $24 \mathrm{~h}$. Wounds were made (magnification, x200). (C) The OCVAR3 cells were transfected or not with miR-124 mimics or scramble for $24 \mathrm{~h}$ (magnification, x200). Results from Transwell invasion assays are presented. (D) The relative invasion of OCVAR3 cells were transfected or not with miR-124 mimics or scramble for $24 \mathrm{~h}$. (E) The SKOV3 cells were transfected or not with miR-124 mimics or scramble for $24 \mathrm{~h}$ (magnification, x200), Results from Transwell invasion assays are presented. (F) The relative invasion of SKOV3 cells were transfected or not with miR-124 mimics or scramble for $24 \mathrm{~h}$. The data from three in vitro independent experiments are presented $\left({ }^{* *} \mathrm{P}<0.01\right)$. NC, negative control; miR, microRNA.

pathological patterns (Fig. 1). The present study compared the expression levels of 30 clinical tumor tissue samples and 30 non-neoplastic ovarian tissues (Fig. 1C) and revealed that the miR-124 expression level was significantly decreased in the majority of ovarian cancer tissues compared with in the non-neoplastic control tissues (Fig. 1D).

miR-124 suppresses the migration and invasion of ovarian cancer cell lines. To investigate the biological role of miR-124 in ovarian cancer, the present study observed its effect on the migration and invasion of the ovarian cancer SKOV3 and OCVAR3 cell lines by transient transfection with miR-124 mimics, miR-124 mimics scramble or negative control (NC). The wound-healing assay indicated that transfection with miR-124 mimics significantly inhibited migration in both cell lines relative to the scramble control or NC at 24 and $48 \mathrm{~h}$ (Fig. 2A and B). Furthermore, ectopic expression of miR-124 markedly decreased invasion of the cell lines compared with the scramble control or NC (Fig. 2C-F).

PDCD6 is a direct target of miR-124 in ovarian cancer. To investigate the potential targets of miR-124, the present study performed bioinformatics analysis using TargetScan and Pictar (Fig. 1E), which predicted that miR-124 targets the PDCD6 3'-UTR region. To determine whether the 3'-UTR of
PDCD6 mRNA is a direct target of miR-124 in ovarian cancer cells, the wild-type (wt) full-length 3'-UTR of PDCD6 or a mutant $(\mathrm{mt})$ sequence was cloned into a luciferase reporter vector (Fig. 1G). Cells were co-transfected with wt 3'-UTR or mt 3'-UTR vectors and miR-124 mimics. The results indicated that overexpression of miR-124 significantly decreased the luciferase activity of reporter genes with wt 3'-UTR compared with the controls. The luciferase activity for the mt 3'-UTR vector was not affected by transfection with miR-124 (Fig. 1F).

Expression of PDCD6 reversed the miR-124-induced suppression of cellular migration and invasion, and induction of cellular apoptosis. Transfection with miR-124 mimics or pcDNA3.1/vector and miR-124 mimics significantly decreased ovarian cancer cell proliferation and migration compared with the controls (Fig. 3). Conversely, co-transfection with miR-124 mimics or pcDNA3.1/PDCD6 in SKOV3 or OCVAR3 cells induced recovery of cell migration and invasion. To investigate whether miR-124 affects cancer cell migration and invasion through PDCD6, the present study performed rescue experiments by upregulating PDCD6 expression in SKOV3 and OCVAR3 cells using pcDNA3.1/PDCD6. The expression level of PDCD6 mRNA was evaluated by qPCR (Fig. 4A and B). The expression level of miR-124 significantly increased subsequent to transient transfection with miR-124 mimics; however, 


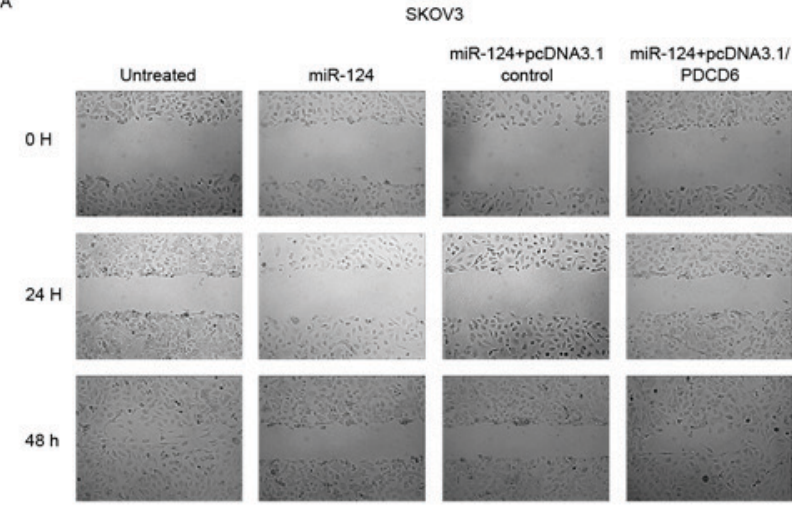

c

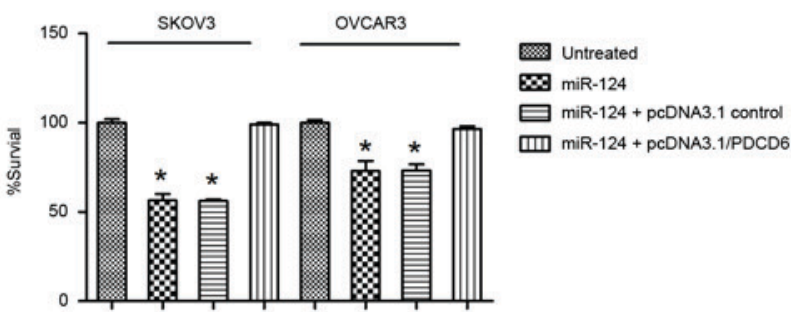

MTT
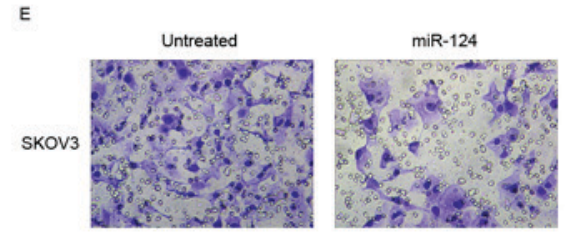

G

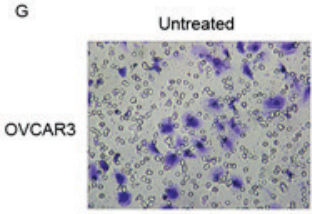

miR-124

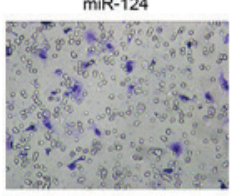

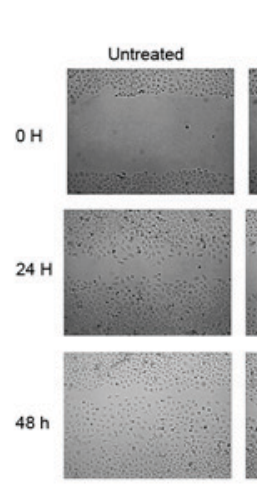

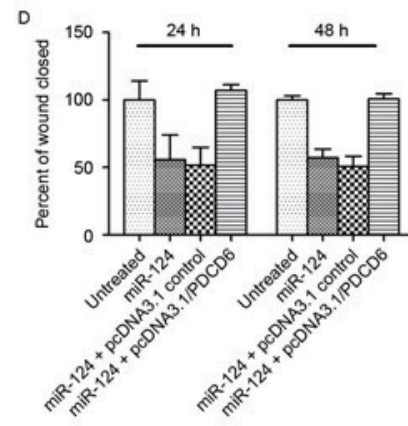

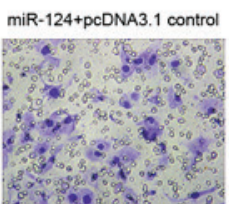
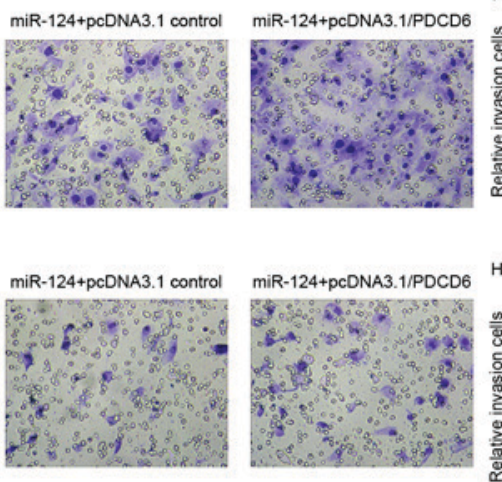
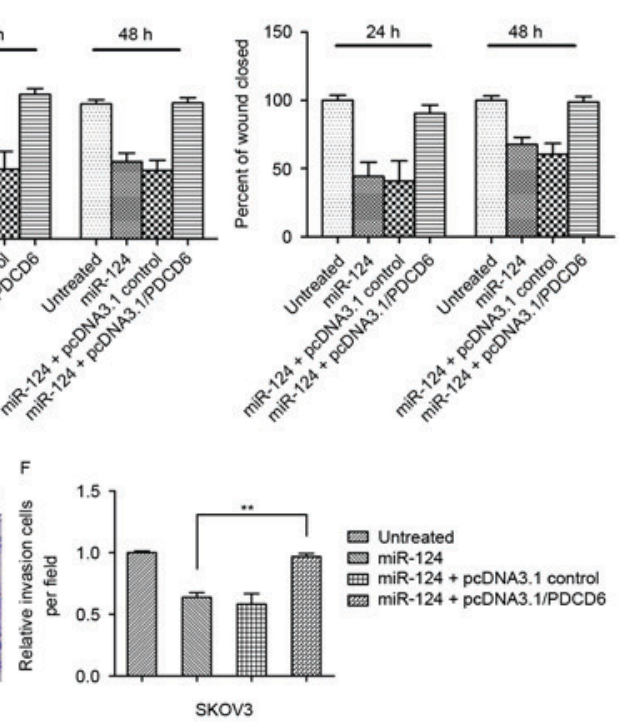

OVCAR3
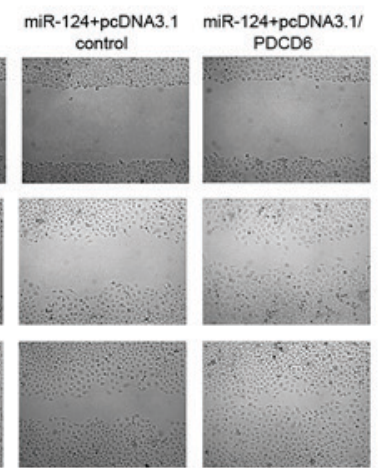

KKOV3

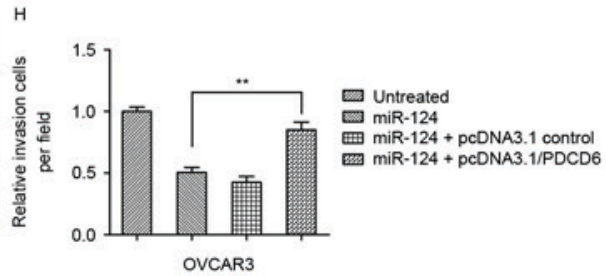

Figure 3. Expression of PDCD6 reversed the miR-124-induced suppression of cellular migration and invasion. (A) Wound-healing assay of SKOV3 cells following co-transfection of NC or miR-124 mimics and either the pcDNA3.1/PDCD6 or pcDNA3.1/vector (magnification, x200). (B) Wound-healing assay of OCVAR3 cells following co-transfection of NC or miR-124 mimics and either the pcDNA3.1/PDCD6 or pcDNA3.1/vector (magnification, x200). (D) Wound-healing assay of representative images are shown at 24 and $48 \mathrm{~h}$. (C) MTT assay of SKOV3 and OCVAR3 cells following co-transfection with NC or miR-124 mimics with either the pcDNA3.1/PDCD6 or pcDNA3.1/vector. The data from three in vitro independent experiments are presented ("P $<0.05)$. (E) Transwell assay of SKOV3 cells following co-transfection of NC or miR-124 mimics and either the pcDNA3.1/PDCD6 or pcDNA3.1/vector (magnification, x200). (F) The relative invasion of SKOV3 cells following co-transfection of NC or miR-124 mimics and either the pcDNA3.1/PDCD6 or pcDNA3.1/vector. (G) Transwell assay of OCVAR3 cells following co-transfection of NC or miR-124 mimics and either the pcDNA3.1/PDCD6 or pcDNA3.1/vector (magnification, x200). (H) The relative invasion of OCVAR3 cells following co-transfection of NC or miR-124 mimics and either the pcDNA3.1/PDCD6 or pcDNA3.1/vector. The data from three in vitro independent experiments are shown $\left.{ }^{* *} \mathrm{P}<0.01\right)$. $\mathrm{NC}$, negative control; miR, microRNA; PDCD6, programmed cell death 6.

the expression level of PDCD6 mRNA markedly decreased, thus miR-124 downregulated PDCD6 mRNA expression level. Transient co-transfection of miR-124 mimics together and pcDNA3.1/PDCD6 rescued the expression level of PDCD6 mRNA. Similar results were obtained by western blot analysis of SKOV3 and OCVAR3 cells co-transfected with miR-124 mimics or NC and pcDNA3.1/PDCD6 or pcDNA3.1/vector (Fig. 4C). The incidence of apoptosis and cell cycle arrest following overexpression of miR-124 and overexpression of PDCD6 were confirmed by flow cytometry. The results demonstrated that transfection of miR-124 mimics markedly increased cell death (Fig. 4D) and the proportion of SKOV3 and OVCAR 3 cells in the $\mathrm{G}_{0} / \mathrm{G}_{1}$ cell cycle phase, but decreased the proportion of cells in S phase (Fig. 4E). However, these effects were reversed by co-transfection of miR-124 mimics and pcDNA3.1/PDCD6.

\section{Discussion}

Currently, the majority of patients with ovarian cancer are diagnosed with distant metastases at an advanced stage, which challenges effective treatment $(9,11,12,23)$. Therefore, more research into potential therapeutic strategies for ovarian cancer is required. Previous studies revealed that miRNAs may 
A

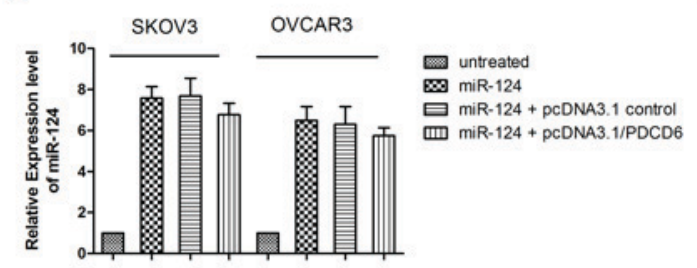

C

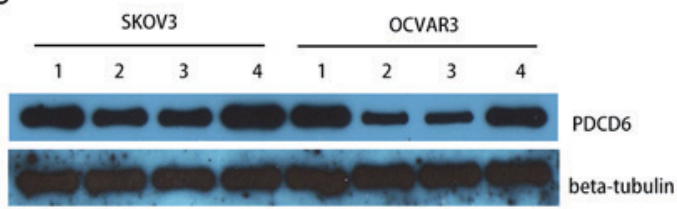

E
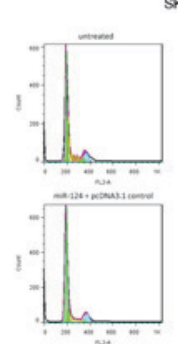

rovs
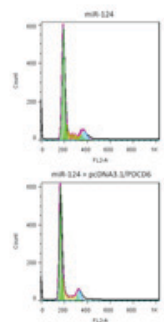

B

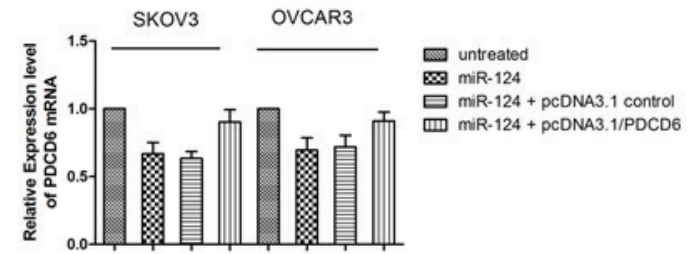

D
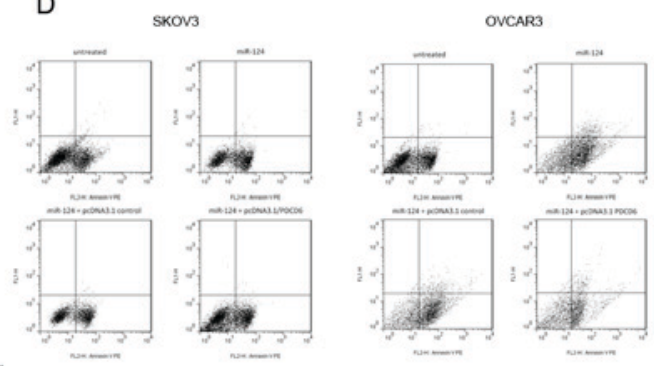

Figure 4. Expression of PDCD6 reversed the miR-124-induced cellular apoptosis. (A) The expression level of miR-124 was determined in SKOV3 and OCVAR3 cells following co-transfection of NC or miR-124 mimics and either the pcDNA3.1/PDCD6 or pcDNA3.1/vector. The expression level of miR-124 markedly increased in cells transfected with miR-124 mimics. Transfection with pcDNA3.1/PDCD6 restored the expression of PDCD6 in SKOV3 and OCVAR3 cells even with co-transfection of miR-124 mimics. (B) The expression levels of PDCD6 were determined in SKOV3 and OCVAR3 cells following co-transfection of NC or miR-124 mimics and either the pcDNA3.1/PDCD6 or pcDNA3.1/vector. The expression level of PDCD6 was markedly suppressed in cells transfected with miR-124 mimics. Transfection of pcDNA3.1/PDCD6 restored the PDCD6 expression level in SKOV3 and OCVAR3 cells even with co-transfection of miR-124 mimics. (C) The expression level of PDCD6 protein was determined in SKOV3 and OCVAR3 following co-transfection with NC or miR-124 mimics and either the pcDNA3.1/PDCD6 or pcDNA3.1/vector. The expression level of PDCD6 protein was suppressed in cells transfected with miR-124. Transfection with pcDNA3.1/PDCD6 restored the expression level of PDCD6 protein in SKOV3 and OCVAR3 cells even with co-transfection of miR-124 mimics. (D) Transfection of miR-124 mimics alone or with pcDNA3.1/vector induced apoptosis of SKOV3 and OCVAR3 cells. (E) The cell cycle status of SKOV3 and OCVAR3 cells following co-transfection of NC or miR-124 mimics and either the pcDNA3.1/PDCD6 or pcDNA3.1/vector was detected by flow cytometry analysis. NC, negative control; miR, microRNA; PDCD6, programmed cell death 6.

regulate migration and invasion, acting as tumor suppressors or oncogenes in human cancer types $(16,17,24,25)$. Further investigation of the roles of miRNAs in cancer development is therefore important for understanding the molecular mechanisms underlying cancer metastasis. Accumulating evidence has suggested miR-124 may function as a tumor suppressor in ovarian cancer (26). The present study revealed that miR-124 was distinctly downregulated in clinical ovarian cancer tissues samples compared with non-neoplastic ovarian tissues from healthy individuals. Furthermore, miR-124 expression level was lower in ovarian cancer lines, exhibiting greater migration and invasion abilities, suggesting that it may be important in ovarian cancer carcinogenesis. Thus, miR-124 appears to be gradually downregulated during ovarian cancer progression, and its upregulation may decrease proliferation and metastasis of ovarian cancer. However, the mechanism underlying miR-124 regulation in ovarian cancer has not been fully elucidated.

PDCD6 is a calcium binding protein in the penta-EF-hand family, and is involved in T-cell receptor-, Fas- and glucocorticoid-induced apoptosis (20,27-30). PDCD6 was identified in rat liver hepatoma and human lung cancer tissues, suggesting that it may be involved in survival pathways, in addition to its pro-apoptotic function (19). Although numerous studies have investigated the association between PDCD6 expression level and disease risk, the results are controversial (19,21,31-34). A previous study indicated that PDCD6 was involved in T-cell receptor-induced apoptosis as a pro-apoptotic factor and that Alix and PDCD6 interaction with pro-caspase- 8 significantly induced cell death via tumor necrosis factor receptor 1 (21). However, another study demonstrated that PDCD6 expression was upregulated in 7,371 tumor tissue samples from lung, breast or colon cancer, which indicated that PDCD6 may be involved in maintenance of cellular viability $(19,35,36)$. Similarly, PDCD6 has been suggested to have a role in cancer cell pathology and contribute to cancer cell viability.

In the present study, PDCD6 was identified as a direct target of miR-124 via luciferase assay and bioinformatics analysis. The present study observed that miR-124 targeted PDCD6 to function as a tumor suppressor in ovarian cancer cells. Furthermore, PDCD6 upregulation rescued the effects of miR-124 overexpression in ovarian cancer lines. These results indicated that the effects of miR-124 on cell proliferation, cell cycle progression, apoptosis, migration and invasion of ovarian cancer cells is partly mediated by inhibiting the expression of PDCD6. 
In future studies, the upstream or downstream modulators of PDCD6 and the effect of miR-124 on signaling pathways of tumor progression by targeting PDCD6 will be investigated. In addition, as chemotherapy resistance is one of the main obstacles in treatment of ovarian cancer, the regulation and function of miR-124 and PDCD6 in chemotherapy resistance in ovarian cancer will continue to be investigated.

In conclusion, the present study provides evidence that the aberrant expression of miR-124 is important in cancer cell migration and invasion. Furthermore, miR-124 induced apoptosis in ovarian cancer cell by regulating PDCD6, a novel and direct target of miR-124. The upregulation of miR-124 markedly inhibited ovarian cancer cell growth, invasion and migration. These results provided a foundation for future studies exploring a new approach to suppressing cancer by targeting miR-124 and suggested that it may provide a novel therapeutic target for ovarian cancer.

\section{References}

1. Farazi TA, Hoell JI, Morozov P and Tuschl T: MicroRNAs in human cancer. Adv Exp Med Biol 774: 1-20, 2013.

2. Lu J, Getz G, Miska EA, Alvarez-Saavedra E, Lamb J, Peck D, Sweet-Cordero A, Ebert BL, Mak RH, Ferrando AA, et al: MicroRNA expression profiles classify human cancers. Nature 435: 834-838, 2005.

3. Iorio MV and Croce CM: MicroRNAs in cancer: Small molecules with a huge impact. J Clin Oncol 27: 5848-5856, 2009.

4. Iorio MV, Visone R, Di Leva G, Donati V, Petrocca F, Casalini P, Taccioli C, Volinia S, Liu CG, Alder H, et al: MicroRNA signatures in human ovarian cancer. Cancer Res 67: 8699-8707, 2007.

5. Abba M, Mudduluru G and Allgayer H: MicroRNAs in cancer: Small molecules, big chances. Anticancer Agents Med Chem 12 733-743, 2012.

6. Gandellini P, Giovannetti E and Nicassio F: MicroRNAs in cancer management: Big challenges for small molecules. Biomed Res Int 2015: 982156, 2015.

7. Calin GA, Sevignani C, Dumitru CD, Hyslop T, Noch E, Yendamuri S, Shimizu M, Rattan S, Bullrich F, Negrini M and Croce CM: Human microRNA genes are frequently located at fragile sites and genomic regions involved in cancers. Proc Natl Acad Sci USA 101: 2999-3004, 2004.

8. Kim K, Zang R, Choi SC, Ryu SY and Kim JW: Current status of gynecological cancer in China. J Gynecol Oncol 20: 72-76, 2009.

9. Lees B and Leath CA III: The impact of diabetes on gynecologic cancer: Current status and future directions. Curr Obstet Gynecol Rep 4: 234-239, 2015

10. Pal MK, Jaiswar SP, Dwivedi VN, Tripathi AK, Dwivedi A and Sankhwar P: MicroRNA: A new and promising potential biomarker for diagnosis and prognosis of ovarian cancer. Cancer Biol Med 12: 328-341, 2015.

11. Langhe R: microRNA and ovarian cancer. Adv Exp Med Biol 889: 119-151, 2015.

12. Maalouf SW, Liu WS and Pate JL: MicroRNA in ovarian function. Cell Tissue Res 363: 7-18, 2016.

13. Zaman MS, Maher DM, Khan S, Jaggi M and Chauhan SC: Current status and implications of microRNAs in ovarian cancer diagnosis and therapy. J Ovarian Res 5: 44, 2012.

14. Zhang Y, Zheng L, Huang J, Gao F, Lin X, He L, Li D, Li Z Ding Y and Chen L: MiR-124 Radiosensitizes human colorecta cancer cells by targeting PRRX1. PLoS One 9: e93917, 2014.

15. Zhang W, Mao YQ, Wang H, Yin WJ, Zhu SX and Wang WC: MiR-124 suppresses cell motility and adhesion by targeting talin 1 in prostate cancer cells. Cancer Cell Int 15: 49, 2015.

16. Lu Y, Yue X, Cui Y, Zhang J and Wang K: MicroRNA-124 suppresses growth of human hepatocellular carcinoma by targeting STAT3. Biochem Biophys Res Commun 441: 873-879, 2013.

17. Zheng YB, Xiao GC, Tong SL, Ding Y, Wang QS, Li SB and Hao ZN: Paeoniflorin inhibits human gastric carcinoma cell proliferation through up-regulation of microRNA-124 and suppression of PI3K/Akt and STAT3 signaling. World J Gastroenterol 21: 7197-7207, 2015.
18. Vito $\mathrm{P}$, Lacanà $\mathrm{E}$ and $\mathrm{D}$ 'Adamio L: Interfering with apoptosis: $\mathrm{Ca}(2+)$-binding protein ALG-2 and Alzheimer's disease gene ALG-3. Science 271: 521-525, 1996.

19. la Cour JM, Høj BR, Mollerup J, Simon R, Sauter G and Berchtold MW: The apoptosis linked gene ALG-2 is dysregulated in tumors of various origin and contributes to cancer cell viability. Mol Oncol 1: 431-439, 2008

20. D'Adamio L, Lacanà E and Vito P: Functional cloning of genes involved in T-cell receptor-induced programmed cell death. Semin Immunol 9: 17-23, 1997.

21. Mahul-Mellier AL, Strappazzon F, Petiot A, Chatellard-Causse C, Torch S, Blot B, Freeman K, Kuhn L, Garin J, Verna JM, et al: Alix and ALG-2 are involved in tumor necrosis factor receptor 1-induced cell death. J Biol Chem 283: 34954-34965, 2008.

22. Giulietti A, Overbergh L, Valckx D, Decallonne B, Bouillon R and Mathieu C: An overview of real-time quantitative PCR: Applications to quantify cytokine gene expression. Methods 25: 386-401, 2001

23. Dwivedi SK, Mustafi SB, Mangala LS, Jiang D, Pradeep S, Rodriguez-Aguayo C, Ling H, Ivan C, Mukherjee $P$, Calin GA, et al: Therapeutic evaluation of microRNA-15a and microRNA-16 in ovarian cancer. Oncotarget 7: 15093-15104, 2016.

24. Cheng CJ, Bahal R, Babar IA, Pincus Z, Barrera F, Liu C, Svoronos A, Braddock DT, Glazer PM, Engelman DM, et al: MicroRNA silencing for cancer therapy targeted to the tumour microenvironment. Nature 518: 107-110, 2015.

25. Maltby S, Plank M, Tay HL, Collison A and Foster PS: Targeting MicroRNA function in respiratory diseases: Mini-review. Front Physiol 7: 21, 2016.

26. Zhang H, Wang Q, Zhao Q and Di W: MiR-124 inhibits the migration and invasion of ovarian cancer cells by targeting SphK1. J Ovarian Res 6: 84, 2013.

27. la Cour JM, Mollerup J, Winding P, Tarabykina S, Sehested M and Berchtold MW: Up-regulation of ALG-2 in hepatomas and lung cancer tissue. Am J Pathol 163: 81-89, 2003.

28. Su D, Xu H, Feng J, Gao Y, Gu L, Ying L, Katsaros D, Yu H, $\mathrm{Xu} \mathrm{S}$ and Qi M: PDCD6 is an independent predictor of progression free survival in epithelial ovarian cancer. J Transl Med 10: 31, 2012.

29. Jung YS, Kim KS, Kim KD, Lim JS, Kim JW and Kim E: Apoptosis-linked gene 2 binds to the death domain of Fas and dissociates from Fas during Fas-mediated apoptosis in Jurkat cells. Biochem Biophys Res Commun 288: 420-426, 2001.

30. Zhou B, Bai P, Xue H, Zhang Z, Shi S, Zhang K, Wang Y, Wang K, Quan Y, Song Y and Zhang L: Single nucleotide polymorphisms in PDCD6 gene are associated with the development of cervical squamous cell carcinoma. Fam Cancer 14: 1-8, 2015.

31. Jang IK, Hu R,LacanáE, D'Adamio L and Gu H: Apoptosis-linked gene 2-deficient mice exhibit normal T-cell development and function. Mol Cell Biol 22: 4094-4100, 2002.

32. Aviel-Ronen S, Coe BP, Lau SK, da Cunha Santos G, Zhu CQ, Strumpf D, Jurisica I, Lam WL and Tsao MS: Genomic markers for malignant progression in pulmonary adenocarcinoma with bronchioloalveolar features. Proc Natl Acad Sci USA 105 10155-10160, 2008

33. Mahul-Mellier AL, Hemming FJ, Blot B, Fraboulet $S$ and Sadoul R: Alix, making a link between apoptosis-linked gene-2, the endosomal sorting complexes required for transport, and neuronal death in vivo. J Neurosci 26: 542-549, 2006.

34. Suzuki K, Dashzeveg N, Lu ZG, Taira N, Miki Y and Yoshida K: Programmed cell death 6, a novel p53-responsive gene, targets to the nucleus in the apoptotic response to DNA damage. Cancer Sci 103: 1788-1794, 2012

35. Høj BR, la Cour JM, Mollerup J and Berchtold MW: ALG-2 knockdown in HeLa cells results in G2/M cell cycle phase accumulation and cell death. Biochem Biophys Res Commun 378: 145-148, 2009.

36. la Cour JM, Mollerup J, Winding P, Tarabykina S, Sehested M and Berchtold MW: Up-regulation of ALG-2 cancer tissue in hepatomas and lung. Am J Pathol 163: 81-89, 2003. 